\title{
Review
}

Xiaoming $\mathrm{Gu}^{*}$

\section{Research progress in urinary tract infection and its therapeutic drugs}

DOI: $10.2478 / \mathrm{ii}-2018-0020$

Received May 02, 2018; accepted May 18, 2018; published online June 29, 2018

\section{Abstract}

Objective: The objective of this study was to understand the pathological mechanism and therapeutic progress in the study of urinary tract infections to provide references for clinical diagnosis and identification and development of therapeutic drugs.

Methods: We summarized the types, pathological mechanisms, and therapeutic drugs for urinary tract infections on the basis of recent publications on these infections, both domestic and abroad.

Results and conclusions: Urinary tract infection is mainly caused by pathogenic bacterial infection and treated by targeting bacterial adhesion, bacterial toxin, protease, urease, and siderophores, as well as using pili as vaccines and small-molecule drugs. Vaccines that target bacterial adhesion can block well the interaction between pathogens and the body, thereby reducing the incidence of urinary tract infections. The clinical efficacy of vaccines targeting bacterial toxins and proteases needs further evaluation. Vaccines targeting iron carriers retard disease progression and attenuate bacterial colonization. Urease-targeted small-molecule drugs exhibit certain curative effects and serious side effects. Small pili-targeted drugs can prevent and treat urinary tract infections by blocking the colonization and invasion of pathogens in animal models of urinary tract infections on the bladder. Adhesive FimH antibodies have entered Phase I clinical trials. However, pilicides, mannosides, and vaccines that target pili, iron carriers, and other virulence factors are still in the experimental or preclinical stages of research.

Keywords: urinary tract infection, urinary tract pathogens, drug therapy, vaccine development

Urinary tract infections caused by pathogens invading the urethra has affected more than 150 million people worldwide. The high recurrence rate and increasingly serious antimicrobial resistance are becoming major burdens on public health [1]. The epidemiological surveys in 2007 showed more than 12 million emergency urinary tract infections in the U.S. annually, causing an economic burden of more than 3.5 billion USD per year [2]. Urinary tract infections, with their severe sequelae (such as pyelonephritis sepsis, infant kidney damage, and premature delivery), have become one of the major diseases that threatens the health of newborns, the elderly, and women.

\section{Pathological mechanism of urinary tract infection}

Urinary tract infections are mainly caused by Gram-positive bacteria, Gram-negative bacteria, and several fungi. The most common pathogen is uropathogenic Escherichia coli (UPEC). Several main pathogens of simple urinary tract infections are UPEC, Klebsiella pneumoniae, Staphylococcus saprophyticus, Enterococcus faecalis, Group B Streptococcus, Proteus, Pseudomonas aeruginosa, Staphylococcus aureus, and Candida [2,3].

Department of Emergency Medicine, Qinzhou TCM Hospital, 262500 Qingzhou, China

*Correspondence: Xiaoming Gu, E-mail: xmgutcm@163.com 
The main pathogens of complex urinary tract infections are UPEC, Enterococci, K. pneumoniae, Candida, $S$. aureus, Proteus, P. aeruginosa, and Group B Streptococcus [4].

According to the infection route, urinary tract infections can be divided into ascending, blood, lymphatic, and direct infections. Ascending infection is the main route of these infections, in which the manure-derived pathogen ascends through the urethra, utilizes flagellum or pili to colonize the urethra, and then migrates to the bladder. Various bacterial adhesins can recognize bladder epithelial cell receptors and mediate colonization. For example, UPEC invades bladder epithelial cells, produces toxins and proteases, and promotes the release of nutrients from the host cells. Moreover, UPEC synthesizes iron carriers for the uptake of iron. After escaping from the body's immune surveillance, pathogens may even migrate through adherents or pili, attach to renal epithelial cells, release tissue-damaging toxins, and pass through the renal tubular epithelial barrier into the blood to cause bacteremia [5].

As a common clinical conditional pathogen, UPEC is also known as the molecular fimbrial chaperone/usher pathway (CUP) [6], and 38 different CUP expression regions have been identified in the gene spectrum of $E$. coli. A single UPEC strain expresses 12 different fimbrial CUPs [7]. In a mouse model, type I pili was found to be necessary for supporting UPEC colonization, invasion, and maintenance of continuous infection in the bladder [8]. After type I pili binds with the FmH adhesion, glycated urinary plaque proteins and $\alpha 1 \beta 3$ integrins can be recognized in three-dimensional structures and then colonize and invade the umbrella cells [9]. Meanwhile, Rho GTPases are activated through signal transduction, rearrange actin and promote the internalization of UPEC [10]. The internalized UPEC can partially escape the body's immune response and resist the treatment of antibacterial drugs. However, during UPEC invasion, the body's innate immunity is protected against UPEC by the urothelial Toll-like receptor (TLR)4 signaling pathway. Bacterial lipopolysaccharide activates the TLR4 pathway to promote cyclic adenosine monophosphate (CAMP) production by adenylyl cyclase 3, while cAMP promotes UPEC vesicles to enter the apical plasma membrane of the umbrella cell through exocytosis. By escaping into the cell membrane, UPEC reverses the expulsion pathway of the body and rapidly proliferates to form transient biofilmlike intracellular bacterial communities (IBCs) [11]. Mature IBCs disperse and invade other cells. Thus, the formation of IBCs has been applied in clinical UPECs. The isolation of this type of invasion and the formation of IBCs allowed UPEC to survive urethral epithelial cells after undergoing several processes such as TLR4-mediated clearance, umbrella cell shedding, urination, and inflammation. In the lower migrating epithelial cells, UPEC can also invade and form quiescent intracellular reserves (QIRs), which are filled with fibrin-type actin between the bio-film and the contained bacteria. Compared with metabolized IBCs, QIRs contain 4-10 non-replicable but viable months and can be reactivated at any time to cause UPEC. This process leads to a recurrence of urinary tract infections [9]. During the differentiation of transitional epithelial cells into umbrella cells, several other signaling pathways may be involved in inducing the release of UPEC in QIRs to the urinary tract. However, the specific mechanism remains to be further explored.

\section{Therapeutic drugs for urinary tract infection}

\subsection{Common clinical drugs for urinary tract infection}

Clinically used drugs for the treatment of urinary tract infections include ciprofloxacin, levofloxacin, nitrofurantoin, sulfamethoxazole, and fosfomycin. However, these drugs induce tendon rupture, hepatotoxicity, pulmonary toxicity, gastrointestinal reactions, skin rashes, allergies, and other adverse reactions. Moreover, antibiotic resistance and high recurrence rate contribute to the incidence of urinary tract infections. Alternative treatments to the existing therapies and alternative treatments to prevent drug resistance are being explored. Numerous clinical or preclinical studies are currently being conducted. 


\subsection{Combination therapy of $\chi$-lactam antibiotics and $\chi$-lactamase inhibitors}

Combination therapies targeting K. pneumoniae carbapenemases enzymes and $\beta$-lactamases for the treatment of urinary tract infections are under clinical study [12]. In in vitro studies, this type of therapy is effective against carbapenem-resistant $E$. coli. The results from clinical trials showed that third-generation cephalosporin ceftazidime combined with $\beta$-lactamase inhibitor avivatan encoded extended-spectrum $\beta$-lactamase and carbapenemase-producing Gram bacteria had good efficacy [13]. However, the mechanism of resistance to $\beta$-lactamase inhibitors in bacteria remains ambiguous. Pathogens of $\beta$-lactamases and carbapenemases are resistant to this combination therapy. A combination of a $\beta$-lactam antibacterial agent and a $\beta$-lactamase inhibitor (BAL30072-BAL29880) is effective for treating various carbapenem-resistant $E$. coli infections. However, K. pneumoniae strains expressing enzymes of $K$. pneumoniae carbapenemases and a plasmid-mediated $\beta$-lactamase (SHV enzyme) are resistant to the complex [14]. Therefore, the combination of $\beta$-lactam antibiotics and $\beta$-lactamase inhibitors should be selected based on the understanding of the specific drug resistance mechanism of the strain.

\subsection{Undertaking drugs}

\subsubsection{Vaccine targeting bacterial adhesion}

Urinary tract infection begins with the adhesion of pathogens. Therefore, the exploration and development of therapeutic drugs for urinary tract infections with bacterial adhesions as a target has become a research hot spot. Previous vaccine studies targeting pili have shown that simply simulating bacterial pili or removing pili from the stimulating organism cannot induce urinary tract infections. The immune response of pathogenic bacteria for the prevention and treatment of urinary tract infections is more difficult to achieve under this pathway. However, the development of vaccines targeting adhesins has shown that Phytophthora sp. can block well the interaction between pathogens and organisms. Langermann et al. [15] studied urinary tract infection models using mice and cynomolgus monkeys and showed that the incidence of urinary tract infections was reduced in individuals immunized with adhesion PapD-PapG or FimC-FimH chaperones. The efficiency of the FimH antibody greatly depended on blocking the number of FimH attachments in the bladder, and the anti-FimH antibody did not affect the balance of $E$. coli in the intestinal microflora. Such vaccines may be structurally modified to enhance their role in stimulating the immune response. For example, the fusion of FimH to the flagellum of FliC triggers a more long-lasting acute immune response through the TLR4/MYD88 pathway [16]. A Phase I clinical study that has been evaluating the effectiveness of FimC-FimH antibody since January has shown that, in addition to the structural modification of FimCFimH, the immune adjuvant used for this antibody is also a synthetic monophosphate A analog that can be used to increase efficacy.

\subsubsection{Vaccines targeting bacterial toxins and proteases}

O'Hanley et al. [17] used a mouse model with pyelonephritis. The results showed that a vaccine targeting the hole-forming toxin (A-type or alpha-hemolysin) of UPEC could delay the progression of renal injury. However, this vaccine did not prevent UPEC colonization in the kidneys. Meanwhile, the vaccine for proteus hemolysin A ( $\mathrm{HpmA}$ ) could not prevent the colonization of bacteria in the kidneys of mouse model with urinary tract infection. However, it targets a vaccine that can damage the bacterial toxin (Pta) of the endothelial cells. Although it has no effect on the bacterial load of the bladder, it can reduce the risk of upper urinary tract infection [18]. Therefore, although hemolysin and protease may provide a good target for the development of vaccines, the clinical efficacy of the vaccine should be further evaluated. 


\subsubsection{Vaccines targeting iron carrier}

Iron ions play a very important role in the adhesion and colonization of urinary tract pathogens (bacteria). The iron content in the bladder is extremely limited, and bacteria rely on iron carriers to collect iron ions to maintain the invasion of the body. This pathological process provides a good entry point for the development of vaccines. Iron carrier and heme expression systems have been found to be increased in animal models and female patients with urinary tract infections [19-21]. This phenomenon facilitates the targeting of the ferric yersiniabactin uptake receptor, heme acquisition protein, and iron uptake transport aerobactin receptor to treat urinary tract infections and provide a theoretical basis for treatment. Targeting ferric yersiniabactin uptake receptor and heme acquisition protein vaccines can prevent disease progression in pyelonephritis mouse model, while vaccines targeting iron uptake transport aerobactin receptor and IreA can attenuate bacterial colonization [21]. These studies confirm the important role of an iron carrier system in the process of urinary tract infections. The result also reflects the different roles of these proteins in the bladder and kidney.

\subsubsection{Small-molecule drugs targeting urease}

Urease plays an important role in the colonization and persistent infection of Proteus and glucococcussaprophyticus. Related studies have focused on acetohydroxamic acid (AHA) using various bacterial ureases to develop more small-molecule inhibitors [22]. AHA has certain therapeutic effect on urinary tract infection caused by urease bacteria and was approved by the US Food and Drug Administration in 1983 to treat urinary tract infections. Nevertheless, AHA's teratogenicity, and may also cause psychological problems and skin mucous membrane damage. Although structurally modified AHA also has good pharmacological activity, the severe side effects remain unsolved. Another urease inhibitor, phosphoramidite, has been demonstrated to be effective in urinary tract infections in animal models, but it presented low stability in the human gastric acid. Low stability leads to poor making of drugs.

\subsubsection{Small-molecule drugs targeting pili}

The small-molecule compounds that target pili are mainly mannosides that inhibit pili function and pilicides that inhibit pilus assembly [23,24]. Pilicides were originally designed as specific inhibitors of UPEC type I pili. However, a broad-spectrum antibacterial activity of pilicides on other bacteria CUP pili was also developed. A single E. coli strain can code for more than 16 different CUP operons. Thus, inhibitors that selectively block pilus assembly have a broad-spectrum antibacterial effect. For example, pilicide ec 240 can block the assembly of type I pili, P pili, and S pili as well as inhibit the movement of flagella [25]. In addition, pilicides have been found to disrupt the biosynthesis of CUP pili against K. pneumoniae and Haemophilus influenzae. However, the pharmacological activity and drugability of pilicides in a mouse model of urinary tract infection caused by simple urinary tract infection and indwelling catheters need to be further studied $[24,26]$. Mannosides can prevent and treat urinary tract infection better and quickly by blocking the colonization and invasion of the pathogen of the urinary tract infection in the bladder as shown using an animal model. Mannosides present high oral bioavailability and good application prospect.

\section{Conclusion}

In summary, the research directions of therapeutic drugs for urinary tract infection are mainly vaccines or small-molecule drugs targeting bacteria, bacterial toxins, proteases, siderophores, urease, and pili. Among these drugs, vaccines with bacterial adhesion as a target can block well the interaction between pathogens and the body, thereby reducing the incidence of urinary tract infections. The clinical efficacy of vaccines 
targeting bacterial toxins and proteases needs further study. Vaccines targeting iron carriers can retard disease progression and attenuate bacterial colonization. Urease as a target of small-molecule drugs not only exhibits certain curative effect but also possess serious side effects. Small-molecule drugs targeting pili can effectively and rapidly prevent and treat urinary tract infections by blocking the colonization and invasion of pathogens to the bladder in animal models of urinary tract infections. Antibodies to FimH have currently entered the Phase I clinical trials. However, pilicides, mannosides, and vaccines targeting pili, siderophores, and other virulence factors are still in experimental or preclinical studies. Moreover, in the development of vaccines, attention should be paid to the impact of vaccines on the normal intestinal microorganisms. Subsequent studies should focus on translating the basic results into clinical trials to explore the feasibility of a new strategy for the treatment of urinary tract infections to provide evidence for its clinical studies.

Acknowledgments: None.

Conflict of interest: The author states no conflicts of interest.

\section{References}

[1] Stamm W.E., Norrby S.R., Urinary tract infections: disease panorama and challenges, J. Infect. Dis., 2001, 183(Suppl. 1), S1-S4.

[2] Foxman B., Urinary tract infection syndromes: occurrence, recurrence, bacteriology, risk factors, and disease burden, Infect. Dis. Clin. North Am., 2014, 28(1), 1-13.

[3] Nielubowicz G.R., Mobley H.L., Host-pathogen interactions in urinary tract infection, Nat. Rev. Urol., 2010, 7(8), 430-441.

[4] Lichtenberger P., Hooton T.M., Complicated urinary tract infections, Curr. Infect. Dis. Rep., 2008, 10(6), $499-504$.

[5] Flores-Mireles A.L., Walker J.N., Caparon M., Hultgren S.J., Urinary tract infections: epidemiology, mechanisms of infection and treatment options, Nat. Rev. Microbiol., 2015, 13(5), 269-284.

[6] Waksman G., Hultgren S.J., Structural biology of the chaperone:usher pathway of pilus biogenesis, Nat. Rev. Microbiol., 2009, 7(11), 765-774.

[7] Wurpel D.J., Beatson S.A., Totsika M., Petty N.K., Schembri M.A., Chaperone: usher fimbriae of Escherichia coli, Plos One, 2013, 8(1), e52835.

[8] Hadjifrangiskou M., Gu A.P., Pinkner J.S., Kostakiotia M., Zhanga E.W., Greenea S.E., et al., Transposon mutagenesis identifies uropathogenic Escherichia coli biofilm factors, J. Bacteriol., 2012, 194(22), 6195-6205.

[9] Hannan T.J., Totsika M., Mansfield K.J., Moore K.H., Schembri M.A., Hultgren S.J., Host-pathogen checkpoints and population bottlenecks in persistent and intracellular uropathogenic Escherichia coli bladder infection, FEMS Microbiol. Rev., 2012, 36(3), 616-648.

[10] Martinez J.J., Hultgren S.J., Requirement of Rho-family GT-Pases in the invasion of type 1-piliated uropathogenic Escherichia coli, Cell. Microbiol., 2002, 4(1), 19-28.

[11] Anderson G.G., Palermo J.J., Schilling J.D., Roth R., Heuser J., Hultgren S.J., Intracellular bacterial biofilm-like pods in urinary tract infections, Science, 2003, 301(5629), 105-107.

[12] Zhanel G.G., Lawson C.D., Adam H., Schweizer F., Zelenitsky S., Lagacé-Wiens P.R., et al., Ceftazidime-avibactam: a novel cephalosporin/ $\beta$-lactamase inhibitor combination, Drugs, 2013, 73(2), 159-177.

[13] Livermore D.M., Mushtaq S., Activity of biapenem (RP-X2003) combined with the boronate $\beta$-lactamase inhibitor RPX7009 against carbapenem-resistant Enterobacteriaceae, J. Antimicrob. Chemother., 2013, 68(8), 1825-1831.

[14] Mushtaq S., Woodford N., Hope R., Adkin R., Livermore D.M., Activity of BAL30072 alone or combined with $\beta$-lactamase inhibitors or with meropenem against carbapenem-resistant Enterobacteriaceae and non-fermenters, J. Antimicrob. Chemother., 2013, 68(7), 1601-1608.

[15] Langermann S., Palaszynski S., Barnhart M., Auguste G., Pinkner J.S., Burlein J., et al., Prevention of mucosal Escherichia coli infection by FimH-adhesin-based systemic vaccination, Science, 1997, 276(5312), 607-611.

[16] Savar N.S., Jahanian-Najafabadi A., Mahdavi M., Shokrgozar M.A., Jafari A., Bouzari S., In silico and in vivo studies of truncated forms of flagellin (FliC) of enteroaggregative Escherichia coli fused to FimH from uropathogenic Escherichia coli as a vaccine candidate against urinary tract infections, J. Biotechnol., 2014, 175, 31-37.

[17] O’Hanley P., Lalonde G., Ji G., Alpha-hemolysin contributes to the pathogenicity of piliated digalactoside-binding Escherichia coli in the kidney: efficacy of an alpha-hemolysin vaccine in preventing renal injury in the BALB/c mouse model of pyelonephritis, Infect. Immun., 1991, 59(3), 1153-1161.

[18] Alamuri P., Eaton K.A., Himpsl S.D., Smith S.N., Mobley H.L., Vaccination with proteus toxic agglutinin, a hemolysinindependent cytotoxin in vivo, protects against Proteus mirabilis urinary tract infection, Infect. Immun., 2009, 77(2), 632-641. 
[19] Watts R.E., Totsika M., Challinor V.L., Mabbett A.N., Ulett G.C., De Voss JJ, et al., Contribution of siderophore systems to growth and urinary tract colonization of asymptomatic bacteriuria Escherichia coli, Infect. Immun., 2012, 80(1), 333-344.

[20] Himpsl S.D., Pearson M.M., Arewång C.J., Nusca T.D., Sherman D.H., Mobley H.L., Proteobactin and a yersiniabactin-related siderophore mediate iron acquisition in Proteus mirabilis, Mol. Microbiol., 2010, 78(1), 138-157.

[21] Brumbaugh A.R., Smith S.N., Mobley H.L., Immunization with the yersiniabactin receptor, FyuA, protects against pyelonephritis in a murine model of urinary tract infection, Infect. Immun., 2013, 81(9), 3309-3316.

[22] Kosikowska P., Berlicki $t$, Urease inhibitors as potential drugs for gastric and urinary tract infections: a patent review, Exp. Opin. Ther. Pat., 2011, 21(6), 945-957.

[23] Piatek R., Zalewska-Piatek B., Dzierzbicka K., Makowiec S., Pilipczuk J., Szemiako K., et al., Pilicides inhibit the FGL chaperone/usher assisted biogenesis of the Dr fimbrial polyadhesin from uropathogenic Escherichia coli, BMC Microbiol., 2013, 13, 131, DOI: 10.1186/1471-2180-13-131.

[24] Cusumano C.K., Pinkner J.S., Han Z., Greene S.E., Ford B.A., Crowley J.R., et al., Treatment and prevention of urinary tract infection with orally active FimH inhibitors, Sci. Transl. Med., 2011, 3(109), 109-115.

[25] Greene S.E., Pinkner J.S., Chorell E., Dodson K.W., Shaffer C.L., Conover M.S., et al., Pilicide ec240 disrupts virulence circuits in uropathogenic Escherichia coli, MBio, 2014, 5(6), e02038.

[26] Klein T., Abgottspon D., Wittwer M., Rabbani S., Herold J., Jiang X., et al., FimH antagonists for the oral treatment of urinary tract infections: from design and synthesis to in vitro and in vivo evaluation, J. Med. Chem., 2010, 53(24), 8627-8641. 